\title{
STATIONARY SETS AND DETERMINING SETS FOR CERTAIN CLASSES OF DARBOUX FUNCTIONS
}

\author{
A. M. BRUCKNER ${ }^{1}$ AND JOHN L. LEONARD ${ }^{2}$
}

1. Introduction. Let $\mathfrak{C}$ be a class of real valued functions defined on an interval $[a, b]$. A set $E$ is called stationary for $\mathcal{e}$ provided any member of $\mathcal{C}$ which is constant on $E$ must be constant on all of $[a, b]$. Several recent articles have been concerned with the problem of determining the stationary sets for various classes of functions [1], [2], [5], [6], [7].

Let us denote by $S(\mathfrak{e})$ the collection of stationary sets for the class e. It is clear that if $\mathfrak{e}_{2} \supset \mathfrak{C}_{1}$ then $S\left(\mathfrak{C}_{1}\right) \supset S\left(\mathfrak{C}_{2}\right)$. Consider now the chain

$$
D \supset D M \supset D B \supset D B_{1} \supset D \mathscr{L} \supset \mathbb{L}
$$

in which $D$ denotes the class of Darboux functions, DMT the class of Darboux measurable functions, $D B$ the class of Darboux Baire functions, $D B_{1}$ the class of Darboux Baire class 1 functions, $D \mathfrak{L}$ the class of Darboux lower semicontinuous functions, and $Q \&$ the class of approximately continuous lower semicontinuous functions. The collections $S(D)$ and $S(\mathscr{L})$ have been characterized [1], [8]. In [5], Marcus found sufficient conditions for a set $E$ to be in $S(D B)$ and necessary conditions for a set to be in $S(D \mathfrak{T})$. He posed the problem of characterizing the stationary sets for the classes $D Q$ and DMT. In $\$ 3$ we obtain these characterizations and in the process we characterize the stationary sets for the remaining classes in the above chain. Then in $\$ 4$ we characterize the determining sets for these classes of functions.

2. Preliminaries. We shall be concerned with functions defined on an interval $[a, b]$. If $f$ is such a function and $A$ is a subset of $[a, b]$, then $f(A)$ denotes the set $\{y: f(x)=y$ for some $x \in A\}$. If $g$ is defined on $[a, b]$ and $f$ is defined on $g([a, b]), f \circ g$ denotes the function whose value at $x \in[a, b]$ is $f(g(x))$. By $\sim A$ we mean the complement of $A$ in $[a, b]$, and $m_{i}(A)$ denotes the Lebesgue inner measure of $A$.

In the sequel we shall consider sets $E$ which intersect every nonempty perfect subset of $[a, b]$. This is equivalent to $\sim E$ being totally

Received by the editors July $27,1964$.

1 This author was supported by NSF Grant GP 1592.

2 NSF Cooperative graduate fellow. 
imperfect; that is, to $\sim E$ containing no nonempty perfect subsets. Every totally imperfect set has zero inner measure but there are totally imperfect sets with positive outer measure. In fact, the interval $[a, b]$ can be decomposed into two disjoint totally imperfect sets [5]. Each of these sets obviously has full outer measure.

A Baire function is a function $f$ with the property that for every open set $G$, the set $\{x: f(x) \in G\}$ is a Borel set. A Baire class 1 function is a function which is the limit of a sequence of continuous functions. If $f$ is a function with the property that $f(I)$ is connected for every interval $I \subset[a, b]$, then $f$ is called a Darboux function. It is clear that the derivative of a differentiable function is a DarbouxBaire class 1 function.

The characterizations of $S(D)$ and $S(D \Re)$ assume the validity of the continuum hypothesis.

3. Stationary sets. We turn now to a discussion of the stationary sets for the various classes of functions under consideration. We begin with a general theorem.

THEOREM 1. Let $\mathfrak{e}$ be a class of functions with the properties:

(a) if $f \in \mathfrak{C}$ and $h$ is continuous from $[a, b]$ onto $[a, b]$, then $f \circ h \in \mathfrak{e}$;

(b) there exists a function $g \in \mathcal{C}$ such that $g$ equals a constant $\lambda$ on a dense subset of $[a, b]$ but $g$ is not identically equal to $\lambda$.

Then a necessary condition that a set $E$ be a stationary set for $\mathfrak{C}$ is that $E$ intersect every nonempty perfect subset of $[a, b]$.

Proof. Suppose $E$ fails to intersect the nonempty perfect set $P^{\prime} \subset[a, b]$. Let $P$ be a nonempty nowhere dense subset of $P^{\prime}$. Denote by $N$ the dense set on which a function $g$ of the hypothesis equals $\lambda$ and let $h$ be a continuous function from $[a, b]$ onto $[a, b]$, such that $h(\sim P) \subset N$. This is possible since $N$ contains a denumerable dense subset which is of the same order type as the set of intervals contiguous to $P$. (See Goffman [3, p. 123].) (For example, $h$ may be taken to be an appropriate Cantor-like function, continuous and nondecreasing, which is constant on each interval contiguous to $P$.) Consider now the function $f=g \circ h$. It follows from the hypothesis that $f \in \mathfrak{e}$. If $x \in \sim P$, then $h(x) \in N$, from which it follows that $f(x)=\lambda$. On the other hand, $\sim N$ is nonempty in $[a, b]$ and $h$ is continuous; thus there is an $x \in P$ such that $h(x) \in \sim N$. For such an $x, f(x) \neq \lambda$. The function $f$ establishes the necessity of the condition.

Corollary. A necessary and sufficient condition that a set $E$ be stationary for the class $D \mathscr{L}, D_{B_{1}}$ or $D B$ is that $E$ intersect every nonempty perfect subset of $[a, b]$. 
Proof. Necessity. The class $D \mathscr{L}$ clearly satisfies condition (a) of Theorem 1. A construction ${ }^{3}$ by Croft [3] of a function in $D \mathscr{L}$, equal to zero almost everywhere but not identically zero, shows that (b) is satisfied. Hence the condition is necessary for $\mathfrak{D} \mathscr{L}$, and thus for the larger classes $D B_{1}$ and $D B$.

Sufficiency. The sufficiency of the condition for the class D® was shown in [5]. It therefore follows for the smaller classes $D B_{1}$ and $D \mathcal{L}$.

It is possible for the class $\mathcal{C}$ of Theorem 1 to be much smaller than D\&. Indeed, given any function $g$ satisfying (b), we may generate a smallest such class containing $g$ by taking the composition of $g$ with all continuous functions from $[a, b]$ onto $[a, b]$. Theorem 1 thus provides a necessary and sufficient condition for a set to be stationary for certain classes $\mathcal{C}$ more restricted than $\mathscr{D} \mathfrak{L}$ (the sufficiency being a consequence of the inclusion $\mathfrak{C} \subset \mathfrak{D} \mathfrak{L})$. Furthermore, if $\mathfrak{C} \subset \mathfrak{D} \mathfrak{L}$, then condition (b) must be satisfied in order to have $S(\mathbb{C})=S(D \mathscr{L})$. For if condition (b) is not satisfied then any dense subset of $[a, b]$ is stationary for $\mathfrak{e}$.

It is natural to ask whether there are any "important" classes $\mathfrak{C}$ properly contained in $D \mathscr{L}$ such that $S(\mathcal{C})=S(D \mathscr{L})$. The answer depends, of course, on what classes one considers to be important. A natural candidate for such a class is the family $a \mathfrak{L}$ of approximately continuous lower semicontinuous functions. The collection $S(a \mathscr{L})$ consists of all sets intersecting every set of positive inner measure. (This characterization follows readily from a similar characterization obtained by Marcus in [8]. See also Theorem 3, below.) Thus, under the assumption that the class $a \mathscr{L}$ is the one which most naturally follows the class $D \mathscr{L}$ in our chain, the above question is answered in the negative.

In the other direction, are there any classes $\mathfrak{C}$ properly containing $D B$ such that $S(\mathbb{C})=S(D B)$ ? There are classes $\mathcal{C}$ which satisfy these requirements. For example, addition of a single suitable function to $D B$ gives a larger class $\mathfrak{C}$ such that $S(\mathbb{C})=S(D B)$. However, the natural class to consider here is the class DIT. The following theorem characterizes the stationary sets for DIT.

THEOREM 2. A necessary and sufficient condition that the set $E$ be stationary for the class DIT is that $E$ intersect every nondenumerable measurable set.

Proof. Necessity. Assume that $\sim E$ contains a nondenumerable measurable subset $M$, and let $M_{0}$ be a nondenumerable zero measure subset of $M$. It follows from [1] that there exists a nonconstant Darboux function $f$, with $f$ vanishing on $\sim M_{0}$, and hence on $E$. The func-

\footnotetext{
${ }^{3}$ The authors wish to thank the referee for calling their attention to this example.
} 
tion $f$ is thus equivalent to the zero function and therefore is measurable. This establishes the necessity of the condition.

Sufficiency. Let $E$ be a set which intersects every nondenumerable measurable subset of $[a, b]$ and let $f$ be a function in $D \mathscr{C}$ which equals a constant $\lambda$ on $E$. If for some $x_{0} \in \sim E, f\left(x_{0}\right) \neq \lambda$, then the set $A=\{x: f(x) \neq \lambda\}$ is nondenumerable, since $f$ is a Darboux function. Since $f$ is measurable $A$ is also measurable. But this implies that $A$ intersects $E$, a contradiction.

The condition of measurability in the above theorem cannot be dropped, for there exist nondenumerable sets, all of whose measurable subsets are denumerable or finite $[4$, p. 168]. (This assumes the continuum hypothesis.)

4. Determining sets. A concept closely related to the concept of a stationary set is that of a determining set.

Definition. Let $\mathcal{C}$ be a class of functions defined on $[a, b]$. A set $E$ is called a determining set for $\mathfrak{C}$ provided any two members of $\mathfrak{C}$ which agree on $E$ must agree on all of $[a, b]$.

We denote by $D(\mathcal{e})$ the determining sets for $\mathfrak{C}$. If $\mathfrak{C}_{2} \supset \mathfrak{C}_{1}$ then $D\left(\mathfrak{C}_{1}\right) \supset D\left(\mathfrak{C}_{2}\right)$. It is clear that if $\mathfrak{e}$ contains the constant functions then $D(\mathfrak{e}) \subset S(\mathfrak{C})$, while if $\mathfrak{e}$ is closed under the operation of subtraction then $S(\mathbb{e}) \subset D(\mathcal{C})$. We observe that all of the classes considered in $\$ 3$ contain the constants, but none of these classes is closed under subtraction. We proceed now to the characterization of the determining sets for the classes considered in $\$ 3$.

THEOREM 3. (a) The only determining set for the classes D, DM, DB and $D B_{1}$ is the interval $[a, b]$.

(b) A necessary and sufficient condition that $E$ be determining for $D \mathscr{L}$ is that $E$ intersect every nonempty perfect set.

(c) A necessary and sufficient condition that $E$ be determining for QL $i$ is that $E$ intersect every set of positive inner measure.

Proof. (a) It suffices to observe that if $x_{0} \in[a, b]$ then the functions

$f(x)=\sin \frac{1}{x-x_{0}}, \quad f\left(x_{0}\right)=1 \quad$ and $\quad g(x)=\sin \frac{1}{x-x_{0}}, \quad g\left(x_{0}\right)=0$

are both in $D B_{1}$ and agree except at $x_{0}$.

(b) Necessity follows from the fact that the class $D \mathscr{L}$ contains the constants, and that the condition is necessary for inclusion in the class $S(\mathbb{D} \mathcal{L})$.

Sufficiency. Suppose $f, g \in D \mathfrak{L}$ and for some $x_{0} \in[a, b], f\left(x_{0}\right) \neq g\left(x_{0}\right)$. Assume $f\left(x_{0}\right)>g\left(x_{0}\right)$ and let $3 \epsilon=f\left(x_{0}\right)-g\left(x_{0}\right)$. Now $g$ is lower semi- 
continuous and therefore in Baire class 1 . Since $g$ also satisfies the Darboux condition, there is a perfect nowhere dense set $P$ containing $x_{0}$ such that the restriction of $g$ to $P$ is continuous at $x_{0}[10$, p. 18]. Therefore there exists an interval $(c, d)$ containing $x_{0}$, with $c, d \in \sim P$, such that if $x \in P^{\prime}=P \cap(c, d)$, then $\left|g(x)-g\left(x_{0}\right)\right|<\epsilon$. On the other hand the semicontinuity of $f$ allows us to determine an interval $\left(c^{\prime}, d^{\prime}\right)$ containing $x_{0}$, with $c^{\prime}, d^{\prime} \in \sim P^{\prime}$, on which interval $f(x)>f\left(x_{0}\right)$ - $\epsilon$. On the perfect set $P^{\prime} \cap\left(c^{\prime}, d^{\prime}\right)$ we have $f(x)>f\left(x_{0}\right)-\epsilon>g\left(x_{0}\right)$ $+\epsilon>g(x)$. We have shown that if two functions in $D \mathscr{L}$ disagree at a point $x_{0}$, then they must disagree on a nonempty perfect set. It follows that any set which intersects every nonempty perfect subset of $[a, b]$ is determining for $D \mathcal{L}$.

(c) It was shown in [6] that a set $E$ is determining for the class of bounded functions in $Q \mathcal{L}$ if and only if $E$ intersects every set of positive inner measure.

Since this class is contained in $Q \mathcal{L}$ the necessity of the condition for the class $Q \mathcal{L}$ is established. We turn now to a proof of the sufficiency of the condition. Let $f, g \in Q \mathcal{L}$. For each $N>0$, define a function $f_{N}$ by

$$
f_{N}(x)=\left\{\begin{array}{cl}
N & \text { if } f(x) \geqq N, \\
f(x) & \text { if }-N<f(x)<N, \\
-N & \text { if } f(x) \leqq-N
\end{array}\right.
$$

and define the function $g_{N}$ analogously. The functions $f_{N}$ and $g_{N}$ are bounded functions in $\propto \mathcal{L}$. Now if $f\left(x_{0}\right) \neq g\left(x_{0}\right)$ and

$$
N>\max \left[\left|f\left(x_{0}\right)\right|,\left|g\left(x_{0}\right)\right|\right],
$$

then $f_{N}\left(x_{0}\right) \neq g_{N}\left(x_{0}\right)$. It follows from the above mentioned result of [6] that $\left\{x: f_{N}(x) \neq g_{N}(x)\right\}$ has positive inner measure. But this set is contained in the set $\{x: f(x)=g(x)\}$. The sufficiency is established.

The table below exhibits the characterizations of the stationary and determining sets for the function classes under consideration.

e

$S(\mathfrak{C})$

$D(\mathcal{C})$

D intersects every nondenumerable set

DIT intersects every nondenumerable measurable set

$D B$ intersects every nonempty perfect set

$D B_{1}$ intersects every nonempty perfect set

$[a, b]$

$[a, b]$

$[a, b]$

$[a, b]$

D\& intersects every nonempty perfect set

intersects every nonempty perfect set

QL intersects every set of positive inner measure intersects every set of positive inner measure 
5. A comparison. We mentioned in $\$ 1$ that every derivative is a Darboux function of Baire class 1 . The converse is not valid. In order to give some sort of measure of how much more restrictive a condition it is for a function to be a derivative than it is for a function to be a Darboux function of Baire class 1, several interesting comparisons between these two classes of functions have been advanced [9], [12], [13]. S. Marcus proved [6] that a necessary and sufficient condition that $E$ be stationary or determining for the class of (finite) derivatives is that $E$ intersect every set of positive inner measure. This result, together with the results appearing in the row labeled by " $D B_{1}$ " in the table above, provides still another comparison of the two classes.

\section{REFERENCES}

1. N. Boboc and S. Marcus, Sur la détermination d'une fonction par les valeurs prises sur un certain ensemble, Ann. Sci. École Norm. Sup. (3) 76 (1959), 151-159.

2. A. Bruckner, Stationary sets for certain classes of derivates of Darboux functions, Michigan Math. J. 11 (1964), 305-309.

3. H. T. Croft, $A$ note on Darboux continuous functions, J. London Math. Soc. 38 (1963), 9-10.

4. C. Goffman, Real functions, Rinehart, New York, 1960.

5. S. Marcus, Les ensembles stationnaires de certaines classes de fonctions, C. R. Acad. Sci. Paris 254 (1962), 1186-1188.

6. - Sur une généralisation de la notion de quasi-analyticitê, C. R. Acad. Sci. Paris 254 (1962), 985-987.

7. - Les ensembles stationnaires de certaines classes de fonctions dérivees, Atti Accad. Naz. Lincei Rend. Cl. Sci. Fis. Mat. Nat. 32 (1962), 484-487.

8. - Private communication dated July 2, 1964.

9. I. Maximoff, On continuous transformation of some functions into an ordinary derivative, Ann. Scuola Norm. Sup. Pisa. 12 (1943), 147-160.

10. - Sur les fonctions ayant la propriêté de Darboux, Prace Math.-Fiz. 43 (1936), 241-265. 1960.

11. I. Natanson, Theory of functions of a real variable, Vol. 2, Ungar, New York,

12. C. J. Neugebauer, Darboux functions of Baire class one and derivatives, Proc. Amer. Math. Soc. 13 (1962), 838-843.

13. Z. Zahorski, Sur la première dérivée, Trans. Amer. Math. Soc. 69 (1950), 1-54.

University of California, Santa Barbara 